\title{
Extreme Emission Line Outflows in the GPS Source 4C 12.50 (PKS 1345+12)
}

\author{
J. Holt ${ }^{1}$, C. N. Tadhunter ${ }^{1}$ and R. Morganti ${ }^{2}$ \\ ${ }^{1}$ The University of Sheffield, Hicks Building, Hounsfield Road, Sheffield, S3 7RH, UK \\ j.holt@sheffield.ac.uk \\ c.tadhunter@sheffield.ac.uk \\ ${ }^{2}$ ASTRON, PO Box 2, 7990 AA Dwingeloo, The Netherlands \\ morganti@astron.nl
}

Received 2002 June 30, accepted 2002 October 4

\begin{abstract}
We present high resolution spectra $(0.7 \AA /$ pix $)$ of the GPS source $4 \mathrm{C} 12.50$ with large spectral coverage $(\sim 4500 \AA)$ taken with the $4.2 \mathrm{~m}$ William Herschel Telescope, La Palma. The slit was aligned along PA $160^{\circ}$ to include the nucleus and emission line region to the NW. An asymmetric halo extending $20 \mathrm{kpc} \mathrm{NW}$ and $12 \mathrm{kpc}$ SE from the nucleus is clearly seen. At the position of the nucleus we observe unusually broad forbidden emission line components (broadest component: FWHM $\sim 2000 \mathrm{~km} \mathrm{~s}^{-1}$ ), blue shifted by up to $2000 \mathrm{~km} \mathrm{~s}^{-1}$ with respect to the halo of the galaxy and $\mathrm{HI}$ absorption. We interpret this as material in outflow. We measure $\mathrm{E}(\mathrm{B}-\mathrm{V})=1.44$ for the broadest, most kinematically disturbed component, corresponding to an actual $\mathrm{H} \beta$ flux 130 times brighter than that measured. We calculate an upper limit for the mass of the line emitting gas of order $10^{6} \mathrm{M}_{\odot}$ for both the intermediate and broad components. Our results are consistent with $4 \mathrm{C} 12.50$ being a young radio source.
\end{abstract}

Keywords: ISM: jets and outflows - ISM: kinematics and dynamics — galaxies: active — galaxies: ISM — galaxies: kinematics and dynamics — galaxies: individual (4C 12.50, PKS 1345+12)

\section{Introduction}

The narrow line region (NLR $<5 \mathrm{kpc}$ ) is an important probe of the ISM close to the central engine in radio galaxies (Tadhunter et al. 2001).

Powerful extended radio galaxies have kinematics generally consistent with gravitational motions in the bulges of the host galaxies (e.g. Heckman, Miley, \& Green 1984). In contrast, GPS radio sources $(<1 \mathrm{kpc}$ in size) show evidence for kinematic disturbance (Gelderman \& Whittle 1994). Highly complex broad emission lines are observed but the nature of the emission line kinematics is still not understood. Asymmetries are believed to be the spectral signatures of systematic flows, but their direction (outflow or inflow) is still under dispute due to the lack of understanding of the dust distribution in the NLR, and lack of accurate host galaxy (rest frame) redshifts.

Compact radio sources are thought to be either young radio sources (e.g. Fanti et al. 1995) or intrinsically compact objects confined by an unusually dense ISM (the 'frustration theory', e.g. van Breugel 1984). High reddening is expected in both scenarios - the nucleus is likely to be enshrouded by large amounts of gas and dust left over from the event(s) which triggered the activity (e.g. a merger, Heckman et al. 1986). With evolution, material along the radio axis will be dissipated by jet-cloud interactions and/or quasar-induced winds (Tadhunter et al. 2001). Eventually cavities will be hollowed out on either side of the nucleus, as is seen in the powerful extended radio source Cygnus A (Tadhunter et al. 1999).

\section{Previous Observations}

4C 12.50 has a double nucleus and shows clear evidence for distorted morphology in the ionised gas: high resolution HST images reveal an arc-like structure 1.2 arcsec $(2.8 \mathrm{kpc}) \mathrm{N}$ of the nucleus and fainter diffuse emission $2 \operatorname{arcsec}(4.7 \mathrm{kpc}) \mathrm{N}$ of the nucleus (see Figure 1). The radio source has distorted, triple morphology aligned along PA $160^{\circ}$ with emission confined to a region $<0.1$ arcsec $(\sim 240 \mathrm{pc})$ in size (see Figure 1). A bright knot towards the northern limit of the jet is tentatively identified as the core (Stanghellini et al. 1997). The jet extends 0.04 arcsec $(95 \mathrm{pc})$ to the SE before bending and expanding into a diffuse lobe. Weak emission extends up to $0.03 \operatorname{arcsec}(70 \mathrm{pc}) \mathrm{NW}$ of the core $\left(H_{0}=75 \mathrm{~km} \mathrm{~s}^{-1}\right.$, $\left.q_{0}=0.0\right)$.

\section{Results}

These preliminary results are based on new, high resolution spectroscopic observations $(\sim 0.7 \AA /$ pix $)$ with large spectral coverage $(\sim 4500 \AA)$ taken in 2001 May with the $4.2 \mathrm{~m}$ William Herschel Telescope on La Palma. For all observations, the slit width was 1.3 arcsec and the seeing was $\sim 1.1$ arcsec (FWHM) or better. For a complete analysis and discussion of PKS 1345+12, see J. Holt et al., MNRAS, submitted.

\subsection{Modelling the Emission Lines}

The emission lines were modelled using the Starlink package DIPSO constraining, for example, the intensity 


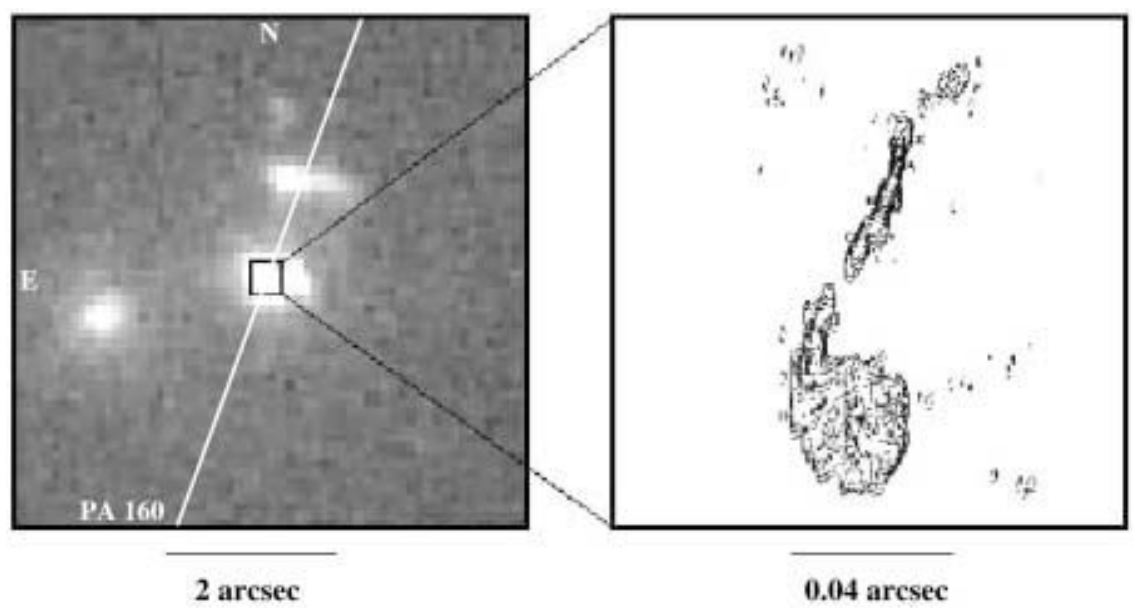

Figure 1 High contrast HST WFPC2 image in [O III] from Axon et al. (2000) with the VLA radio map (5 GHz) from Stanghellini et al. (1997). For a higher resolution map, refer to the original publication.

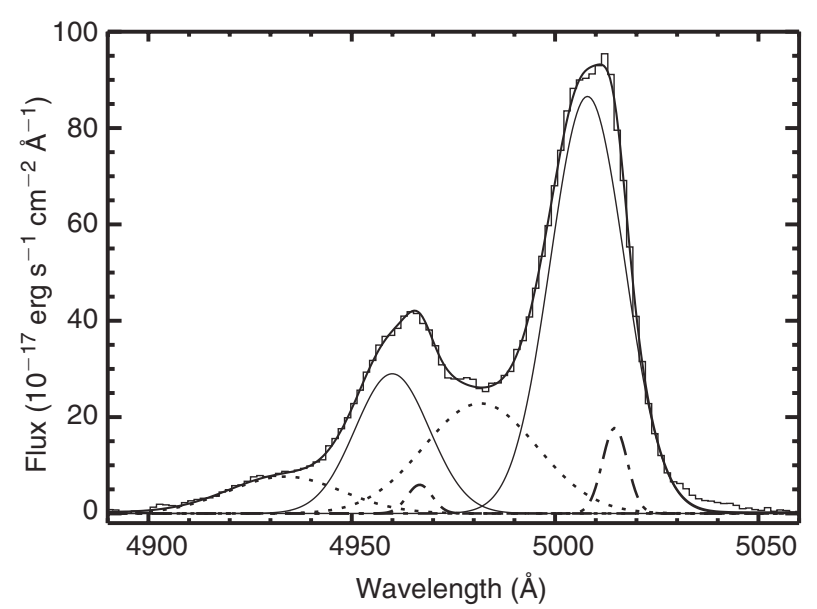

Figure 2 Model for [O III] $\lambda \lambda 4959,5007$ (bold line) comprising 3 components for each line: narrow (dot-dashed line), intermediate (solid line) and broad (dotted line).

ratios and shifts between doublet lines, where possible, in accordance with atomic physics. In order to model the unusually broad $[\mathrm{O}$ III] $\lambda \lambda 4959,5007$ emission lines in the nucleus it is essential to use three Gaussians (see Figure 2). These three components represent line emission from three distinct redshift systems:

(1) A narrow component ( $\mathrm{FWHM} \sim 360 \mathrm{~km} \mathrm{~s}^{-1}$ ) assumed to emanate from the ambient, quiescent ISM. Its velocity is consistent with the deep HI $21 \mathrm{~cm}$ absorption (Mirabel 1989; see also Morganti et al. these proceedings), and the extended [O II] halo.

(2) An intermediate component (FWHM $\sim 1260 \mathrm{~km} \mathrm{~s}^{-1}$ ) blueshifted by $\sim 400 \mathrm{~km} \mathrm{~s}^{-1}$ relative to the narrow component.

(3) A broad component (FWHM $\sim 1960 \mathrm{~km} \mathrm{~s}^{-1}$ ) blueshifted by $\sim 1980 \mathrm{~km} \mathrm{~s}^{-1}$ relative to the narrow component.

The velocity shifts in the [O III] lines clearly indicate that they originate from three distinct regions along the

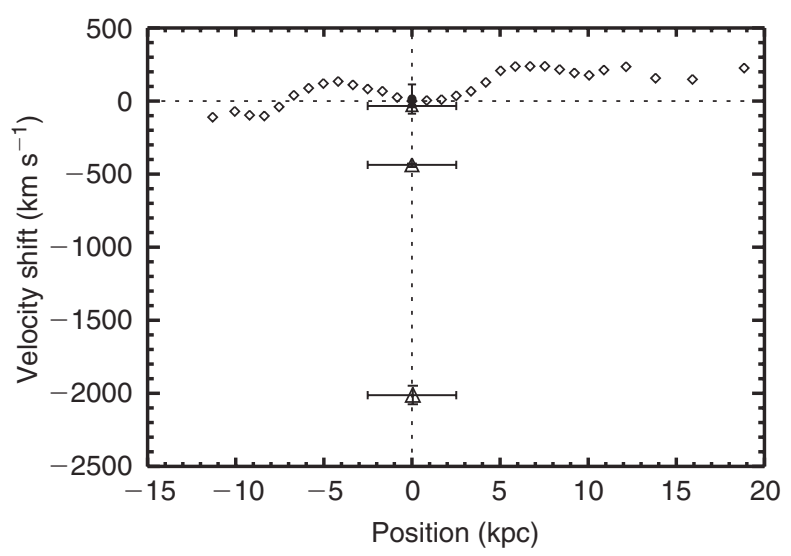

Figure 3 Comparison of the three components of [O III] (open triangles) with the spatially extended [O II] $\lambda \lambda 3726$, 3729 emission (open diamonds) and the deep HI $21 \mathrm{~cm}$ absorption from Mirabel (1989) (filled circle) provides a good estimate of the galaxy rest frame. Two of the $[\mathrm{O} \mathrm{III}]$ components trace material in outflow.

line of sight. The narrowest component of [O III] is consistent with the spatially extended [O II] emission and $\mathrm{HI}$ absorption and represents the galaxy rest frame. Hence, the intermediate and broad components of [O III] trace material blueshifted with respect to the galaxy system, suggesting that the ISM emitting these components is in outflow (see Figure 3).

\subsection{Evidence for High Reddening in the Nucleus}

We measure $\mathrm{E}(\mathrm{B}-\mathrm{V})$ values, calculated from the $\mathrm{H} \alpha / \mathrm{H} \beta$ ratio, of $0.06,0.42$, and 1.44 for the narrow, intermediate, and broad components, respectively. The broad component is highly reddened and corrections for reddening yield $\mathrm{H} \beta$ fluxes a factor of 130 brighter than those measured. This reddening is consistent with $\mathrm{Pa} \alpha / \mathrm{H} \alpha$ ratios estimated using Pa $\alpha$ measurements from Veilleux, Sanders, \& Kim (1997). Note that the highly broadened $\mathrm{Pa} \alpha$ component detected by Veilleux et al. (1997) is not associated with a classical broad line region (BLR) as it is also observed in 
the forbidden lines and therefore represents a broadened NLR component.

\subsection{Estimating the Gas Mass}

Emission line luminosities are related to the mass of line emitting gas by

$$
M_{\mathrm{gas}}=m_{\mathrm{p}} \frac{L(\mathrm{H} \beta)}{N_{\mathrm{e}} \alpha_{\mathrm{H} \beta}^{\mathrm{eff}} \mathrm{h} \nu_{\mathrm{H} \beta}}
$$

where $N_{\mathrm{e}}$ is the electron density $\left(\mathrm{cm}^{-3}\right) ; m_{\mathrm{p}}$ is the mass of the proton $(\mathrm{kg}) ; L(\mathrm{H} \beta)$ is the luminosity of the $\mathrm{H} \beta$ line: $0.44 \pm 0.04,5.50 \pm 0.20$, and $26.59 \pm 0.59\left(10^{40} \mathrm{erg} \mathrm{s}^{-1}\right)$ for the narrow, intermediate, and broad components, respectively; $\alpha_{\mathrm{H} \beta}^{\mathrm{eff}}$ is the effective recombination coefficient for $\mathrm{H} \beta\left(\mathrm{cm}^{3} \mathrm{~s}^{-1}\right.$; see Osterbrock 1989); and $\mathrm{h} \nu_{\mathrm{H} \beta}$ is the energy of the $\mathrm{H} \beta$ photons (erg).

The density diagnostic, the $[\mathrm{S}$ II $] \lambda \lambda 6716,6730$ doublet, can only be modelled with very high densities for the intermediate and broad components. We estimate lower limits for the density of the intermediate and broad components of $\sim 4000 \mathrm{~cm}^{-3}$. These lead to an upper limit for the total mass of the line emitting gas of $9.17 \times 10^{5} \mathrm{M}_{\odot}$.

\section{Conclusions}

Our data show

- complex broad emission lines;

- blue shifts up to $\sim 2000 \mathrm{~km} \mathrm{~s}^{-1}$ with respect to the galaxy halo and HI absorption;

- large reddening (broadest component $\mathrm{E}(\mathrm{B}-\mathrm{V}) \sim 1.44$ );

- high densities and high ionisation for the intermediate and broad components.

For the blueshifted components we calculate

- a lower limit for the density;

- an upper limit for the gas mass of order $10^{6} \mathrm{M}_{\odot}$.

Our results are consistent with the model proposed for the compact flat spectrum radio source PKS 1549-79 by Tadhunter et al. (2001) in which the nucleus is cocooned in gas and dust in the early stages and drives outflows which will eventually hollow out the ionisation cones on either side of the nucleus (see Figure 4).

Our estimates for the mass of emitting gas of order $10^{6} \mathrm{M}_{\odot}$ are also consistent with the radio source being young. Using the equations presented by O'Dea (1998) from de Young (1993), a smooth medium with the density we measure would require gas masses of order $10^{8}-$ $10^{10} \mathrm{M}_{\odot}$ to frustrate the radio source - much larger than we estimate for the blueshifted components.

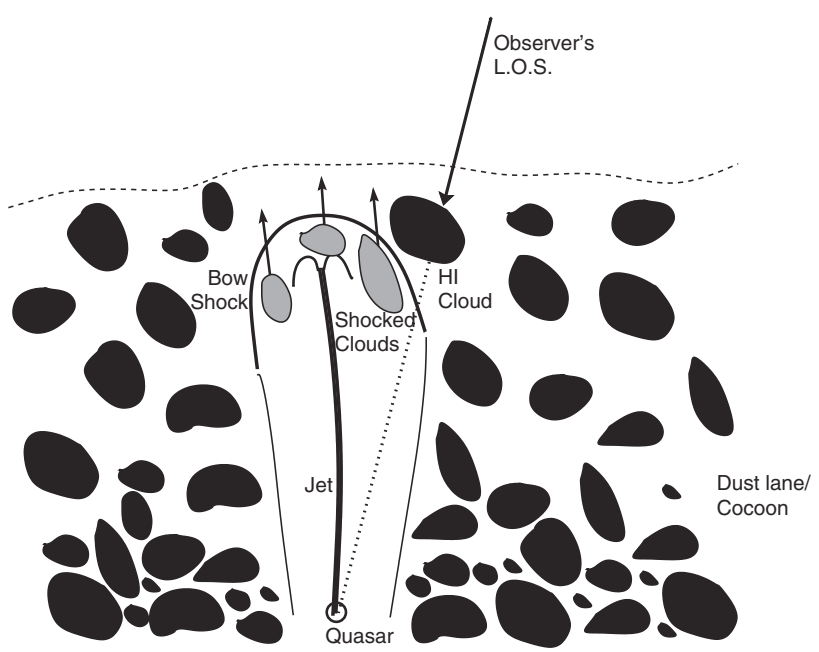

Figure 4 Model proposed for the compact flat spectrum radio source PKS 1549-79 by Tadhunter et al. (2001).

\section{Acknowledgments}

JH acknowledges a PPARC PhD studentship. The William Herschel Telescope is operated on the island of La Palma by the Isaac Newton Group in the Spanish Observatorio del Roque de los Muchachos of the Instituto de Astrofisica de Canarias.

\section{References}

Axon, D. J., Capetti, A., Fanti, R., Morganti, R., Robinson, A., \& Spencer, R. 2000, AJ, 120, 2284

de Young, D. S. 1993, ApJ, 402, 95

Fanti, C., Fanti, R., Schilizzi, R. T., Spencer, R. E., \& Stanghellini, C. 1995, A\&A, 302, 317

Gelderman, R., \& Whittle, M. 1994, ApJS, 91, 491

Heckman, T. M., Miley, G. K., \& Green, R. F. 1984, ApJ, 281,525

Heckman, T. M., Smith, E. P., Baum, S. A., van Breugel, W. J. M., Miley, G. K., Illingworth, G. D., Bothun, G. D., \& Balick, B. 1986, ApJ, 311, 526

Mirabel, I. F. 1989, ApJ, 340, L13

O’Dea, C. P. 1998, PASP, 110, 493

Osterbrock, D. E. 1989, Astrophysics of Gaseous Nebulae and Active Galactic Nuclei (Mill Valley, California: University Science Books)

Stanghellini, C., O'Dea, C. P., Baum, S. A., Dallacasa, D., Fanti, R., \& Fanti, C. 1997, A\&A, 325, 943

Tadhunter, C. N., Wills, K., Morganti, R., Oosterloo, T., \& Dickson, R. 2001, MNRAS, 327, 227

Tadhunter, C. N., Packham, C., Axon, D. J., Robinson, A., Hough, J., Young, S., \& Sparks, W. 1999, ApJ, 512, L91

van Breugel, W. J. M. 1984, in VLBI and Compact Radio Sources, IAU Symposium 110, ed. R. Fanti, K. Kellermann, \& G. Setti (Dordrecht: Reidel), 59

Veilleux, S., Sanders, D. B., \& Kim, D. C. 1997, ApJ, 484, 92 